\title{
A systems engineering framework for the design of bioprocess operator training simulators
}

\author{
Joseph Isimite ${ }^{1}$, Frank Baganz ${ }^{1}$, Volker Hass ${ }^{1,2}$ \\ ${ }^{1}$ Department of Biochemical Engineering, University College London, Gordon Street, London, WC1H 0AH \\ ${ }^{2}$ Furtwangen University, Jakob-Kienzle-Str. 17, D-78054 Villingen-Schwenningen, Germany
}

\begin{abstract}
Operator training simulators (OTS) are widely used in several industries including chemical processing, oil and gas, medicine, aircraft and nuclear facilities. However, developing a biorefinery OTS is a complex engineering design activity that requires a structured technique. This paper presents a structured methodology that applies design frameworks from other disciplines and a user-centred approach for biorefinery OTS design. These include the definition of end user requirements (operator training needs), and the analysis of these requirements using Quality Function Deployment (QFD). Furthermore, an algorithm for bioprocess optimisation and automatic adjustment of operating parameters is developed for integration into the OTS. This algorithm is based on the Nelder-Mead simplex method for multi-dimensional function minimisation.

Identified user requirements were categorized into primary, secondary and tertiary training needs, with increasing levels of detail from primary to tertiary needs. The relationships between identified operator training needs and OTS technical and functional specifications were investigated, and a priority rating assigned to the most important OTS specifications. Identified OTS specifications were evaluated for robustness to ensure that important features were not omitted from the final design.
\end{abstract}

\section{Introduction}

As the search for more efficient ways to produce bioethanol and other biofuels such as biodiesel and biogas intensifies, there is a responsibility to provide fitfor-purpose education for the next generation of bioengineers and the right skill and training for process operators who will run the biorefineries required to meet the energy challenges of today. In addition, increasing production efficiencies in biorefineries will make biofuels economically competitive with more established fossil derived fuels.

Developing a fully functional biorefinery Operator Training Simulator (OTS) is a complex activity, both in terms of the technical requirements and time needed for completion. A systems engineering approach is required to facilitate the OTS development process. This approach ensures that the OTS design activity is completed in a structured and systematic manner. Systems engineering is a methodology that ensures the sound application of scientific and engineering effort to transform clearly identified needs into a set of technical and functional performance parameters for the design activity [1]. The process is usually iterative and involves system definition, synthesis, analysis, design, test and effectiveness evaluation. Another key element of this process is the integration of different subsystems in a manner that ensures compatibility and optimum performance of the final design. Figure 1 is a summary of the systems engineering strategy proposed for biorefinery OTS design.

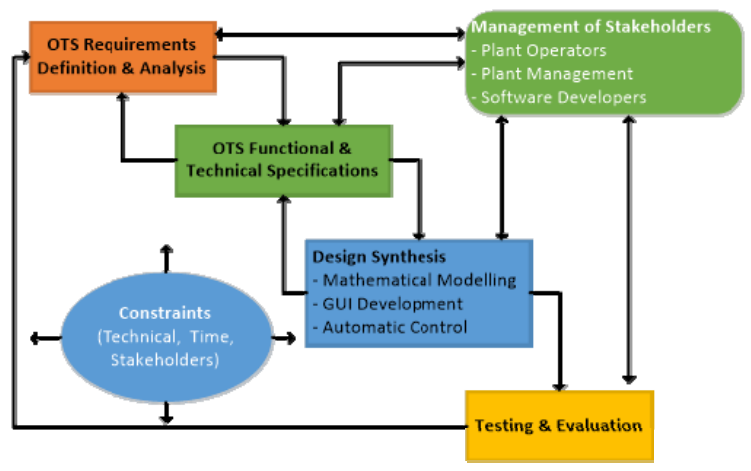

Fig. 1: Systems engineering approach adopted for OTS development (adapted from Buede, 2009)

\section{OTS design methodology}

The systematic OTS design process involves the following steps:

1. Definition of OTS design targets.

2. Identification of target user requirements (training needs analysis).3. Screening of identified user requirements through Quality Function Deployment (QFD).

4.Definition of OTS functional and technical specifications.

\footnotetext{
* Corresponding author: joseph.isimite.15@ucl.ac.uk
} 
5. Staged OTS development, involving model definition, model implementation and development of graphical user interfaces for operator training.

6. Evaluation of operator training effectiveness with developed OTS.

\subsection{Definition of OTS design targets}

An operator training simulator offers several benefits for process operators in an industrial bioprocessing plant. However, because of the constrains inherent in the design process, clearly specified design targets should be identified early in the development process to ensure optimum direction of effort and resources. The following are some examples of OTS design targets.

1. Design to enhance operator competence.

2. Design to improve plant availability \& equipment reliability.

3. Design to improve operational efficiency.

4. OTS design to improve safety (prevent damage to people, plant and the environment)

Operator competence is the result of acquiring the experience and training necessary to properly perform specific tasks within a biorefinery. This experience can be measured in terms of fundamental process knowledge, practical skills acquired through years of practice, and a calm, composed and confident response during emergency situations when added pressure increases the risk of operator error. The level of operator competence has been identified as a major contributing factor in the initiation and escalation of deviations from normal operating conditions [2]. Figure 2 shows the main contributory factors in the evolution of abnormal situations in a process plant, with people and work context responsible for $42 \%$ of incidents.

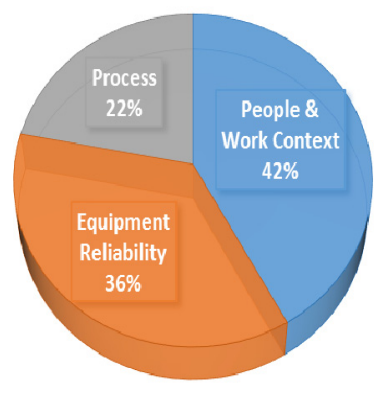

Fig. 2: Major causes of incidents in the process industries

Figure 2 also shows that equipment reliability plays a role in causing incidents. While plant maintenance personnel are primarily responsible for ensuring equipment reliability and minimizing losses due to downtimes, operators play an important role in promoting the reliability management process. Poor understanding and execution of operational strategies may result in operating equipment outside their optimum ranges, leading to equipment damage and loss of production.

In a biorefinery, the optimum transformation of raw materials into final products takes place within a strictly defined operating envelope. It is the duty of the operator to understand this optimum operational window and utilize his knowledge and skills to maximize the input of materials, and control of process parameters through continuous monitoring and observation. Deviations from optimum operating conditions demand that the operator employ effective operational strategies to return the plant to normal operating conditions, and in extreme cases, prevent the escalation of unsafe conditions which may lead to loss of materials, environmental damage, and damage to people or equipment.

\subsection{Identification of target user requirements}

The main target users for the OTS are the control room operators (CROs). Other users include field operators, training managers, and plant engineers. Training needs analysis was selected as the methodology to elicit target user requirements for OTS design. The purpose of the training needs assessment was to identify the precise knowledge, skills and attitudes necessary for biorefinery plant operators to perform their jobs efficiently and effectively.

Several methods are used to collate and analyse information during the training needs analysis process [3]. The methods used in this work include face to face interviews, focus group interviews, and questionnaires. The target groups used to elicit the training needs for OTS design are control room operators, field operators, plant engineers, and training and technical competence assurance staff.

In defining operator training needs, it was important to express them concisely and clearly, as explained below.

Specific: Relates to precise plant or operator performance criteria.

Measurable: Clear metric(s) can be defined to determine if need has been achieved or not.

Achievable: Can be implemented using available tools and resources.

Realistic: Addresses a need that is relevant to commercial biorefineries.

Time-bound: Can be implemented and training effectiveness assessed within three years.

Training needs definition and refinement should be a continuous process, as discussions between the different stakeholders continue to take place.

\subsection{Screening of identified user requirements}

The analysis phase involves the screening of responses received from target users. Quality Function Deployment (QFD) methodology was used to group identified training needs into primary, secondary and tertiary training needs [4]. The primary tier of training needs provides a high-level description of operator training requirements. The primary needs were phrased in line with the four OTS design targets defined in section 2.1. The secondary tier of needs provides more detail about the needs stated in the primary tier while tertiary needs provide the most detail about the training requirements 
identified. The QFD matrix used in this study is shown in Figure 3.

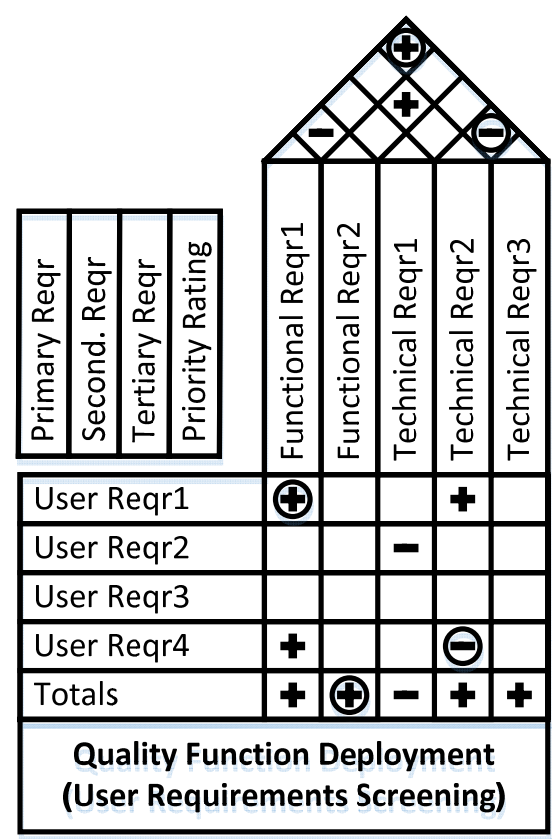

Fig. 3: QFD Matrix (also called House of Quality - HOQ).

A benefit of using the QFD matrix is that it facilitates the participation of all stakeholders in the analysis process. This ensures that the OTS design process takes into account the views of plant operators, plant management, and OTS design team in identifying technical and functional specifications to be included in the OTS. The priority rating shows the degree of importance of each training requirement. Ranking values from $1-10$ are assigned after discussions with all relevant stakeholders - with 10 showing a training requirement that is most important and 1 depicting a training requirement that is least important.

Thirteen (13) training needs were identified in section 2.2 and these were used for QFD analysis. Based on responses from operators and engineers, the five most important training needs for biorefinery OTS design include:

1. Use of OTS to improve liquefaction performance to eliminate fouling problems

2. Effectively manage heat integration in distillation and evaporation sections

3. Effectively complete evaporator change-overs (a non-routine operation)

4. Start-up with minimal number of trips

5. Reduce unplanned downtime due to poor plant operation.

Based on the identified training needs, twenty (20) important OTS specifications were determined as necessary to achieve the training targets. These were categorised into ten functional specifications and ten technical specifications. The relationship matrix in the QFD shows how each OTS technical or functional specification can help in meeting the requirements of each training need by assigning a value to each specification-training need pair.
The correlation matrix at the top of the QFD shows whether there is a positive or negative effect between each pair of specifications.

\section{Results}

From the QFD analysis, the ten most important OTS specifications, in their order of importance include:

1. Alarm annunciation and criticality display.

2. Use of OTS to teach process control principles.

3.Ability to simulate abnormal plant operating conditions.

4. High level of fidelity with plant distillation section.

5.High level of fidelity with plant evaporation section.

6. High level of fidelity with plant liquefaction section.

7.Simulate routine deviations from normal operations. tasks.

8. Train operators to perform complex non-repetitive

9. Test different process optimization strategies.

10. Improve communication between operators.

\section{Discussions}

A structured design approach that utilises systems engineering principles has been used for biorefinery OTS design. The structured approach made it possible to effectively combine and manage the different elements required for successful OTS design. The conceptual framework for the design activity allowed for the complete specification of the different stages of the design process, from user requirement identification, to OTS design and scientific evaluation of operator training effectiveness using the developed OTS. Four OTS design targets were identified. These design targets are not mutually exclusive, as the realisation of one objective helps achieve some or all of the other objectives. More competent operators will be better able to manage and improve plant operational efficiency [5]. They will also make less mistakes that can potentially damage equipment and make the plant unavailable.

Operator training needs were identified for the OTS design through questionnaires and focus group interviews. It was apparent from discussions with biorefinery plant operators that improvements were required in the plant. Operators were convinced that an OTS will be valuable in helping them improve their process knowledge and capability to run the plant efficiently. Managing fouling in downstream processes (distillation and evaporation), and the confidence to carry out difficult non-routine operations such as evaporator change overs, were identified as particular areas that required attention. Operator shift teams also differed significantly in the levels of competence and confidence displayed. It was evident that confidence improved with experience.

An OTS can be used to increase the level of exposure and practice of carrying out potentially demanding tasks, by helping them gain virtual operational experience in a 
relatively short time [6]. Cross learning and interaction between shift teams is a potential area that can be addressed by the OTS. The priorities assigned by engineers for OTS technical and functional specifications agreed with the important needs identified through discussions with plant operators. There is consensus regarding the need to improve operators' ability to handle complex non-routine tasks and improve their overall knowledge of the plant. For example, operators with experience in operating the upstream section of the plant (liquefaction and fermentation) had limited knowledge of downstream sections (distillation and product purification), and vice versa. An OTS will therefore be an important tool for training operators to fully understand the operation of the entire biorefinery.

The QFD methodology identified the most important OTS specifications. Alarm annunciation and criticality display appears to be a significant specification required in the OTS. Alarms help operators to quickly observe deviations from optimum process conditions before they escalate to unacceptable levels that will compromise product quality or the safety of people and plant equipment.

It is also deduced from the QFD analysis that operator understanding, and implementation of process control strategies should be an important functional specification of the OTS. It is important that the OTS can simulate routine and catastrophic deviations from normal operating conditions. The ability to simulate both routine and catastrophic deviations from normal operating conditions should be implicit elements of the developed OTS.

High levels of fidelity are required for some unit operations such as distillation and evaporation. This includes the fidelity of the process models describing the physical and chemical processes occurring in these unit operations, and the look and feel of the graphical user interfaces for these sections of the plant. The process models developed for tank filling, liquid heating, and starch hydrolysis are comparable to and can replicate what happens on the actual plant. The graphical user interfaces are also very similar to those found on the actual plant DCS.

\section{References}

1. D.M. Buede, The engineering design of systems: models and methods. 2nd ed. ,Hoboken, N.J., John Wiley \& Sons (2009).

2. ASM Consortium. Abnormal Situation Management, Available at: https://www.asmconsortium.net/Pages/default.aspx. Accessed Accessed Dec 2016. (2014)

3. C. Sleezer, D.F. Russ-Eft, K. Gupta, A practical guide to needs assessment. 3rd ed. San Francisco, Wiley, (2014).

4. L. Chan, M. Wu, Quality function deployment: A literature review. Eur. J. Oper. Res. 143:463-497. (2002).
5. J. Abel, \& R. Rys, Training simulators smooth operations, Control global, Online, Available at http://www.controlglobal.com/articles/2016/userschime-in-on-benefits-of-operator-trainingsimulators/. (2016).

6. S. Nazir, S. Colombo, D. Manca, Testing and analyzing different training methods for industrial operators: an experimental approach. Comp. A. Chem. Eng. 32:667-672 (2013). 